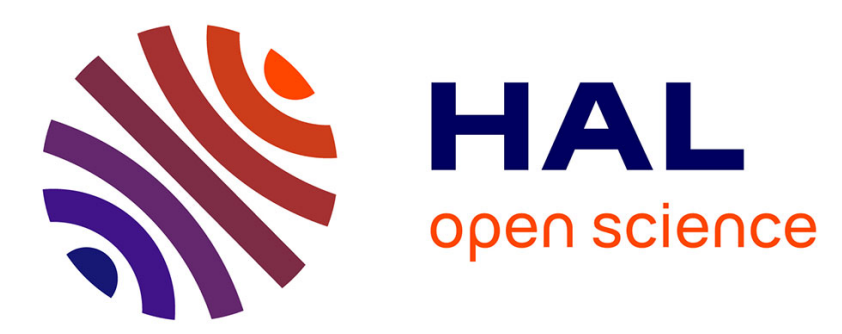

\title{
Reduced simulation model for flow analysis in a sawmill internal supply chain
}

Philippe Thomas, Marie-Christine Suhner, André Thomas

\section{To cite this version:}

Philippe Thomas, Marie-Christine Suhner, André Thomas. Reduced simulation model for flow analysis in a sawmill internal supply chain. International Conference on Industrial Engineering and Systems Management, IESM'15, Oct 2015, Seville, Spain. hal-01225373

\section{HAL Id: hal-01225373 \\ https://hal.science/hal-01225373}

Submitted on 6 Nov 2015

HAL is a multi-disciplinary open access archive for the deposit and dissemination of scientific research documents, whether they are published or not. The documents may come from teaching and research institutions in France or abroad, or from public or private research centers.
L'archive ouverte pluridisciplinaire HAL, est destinée au dépôt et à la diffusion de documents scientifiques de niveau recherche, publiés ou non, émanant des établissements d'enseignement et de recherche français ou étrangers, des laboratoires publics ou privés. 


\title{
Reduced simulation model for flow analysis in a sawmill internal supply chain
}

\author{
(presented at the 6th IESM Conference, October 2015, Seville, Spain) ${ }^{\circ} \mathrm{I}^{4} \mathrm{e}^{2} 2015$
}

\author{
Philippe THOMAS ${ }^{1,2}$, Marie-Christine SUHNER ${ }^{1,2}$, André THOMAS ${ }^{1,2}$ \\ ${ }^{1}$ Université de Lorraine, CRAN, UMR 7039, Campus Sciences, BP 70239, 54506 Vandœuvre-lès-Nancy cedex, France \\ ${ }^{2}$ CNRS, CRAN, UMR7039, France
}

\{philippe.thomas, marie-christine.suhner, andre.thomas\}@univ-lorraine.fr

\begin{abstract}
Accurate and simple simulation flow model is useful to make decision at the right time to manage supply chain or workshop. To do that, different reduction model complexity approaches have been proposed. One of them is to associate discrete event model of bottlenecks and continuous model of other work centers according to the theory of constraints. The continuous approximation is used only do determine how the bottlenecks are fed. Different continuous model have been proposed in the past. This paper focuses on the association of regression trees and neural networks in order to benefit of the advantages of each other. This approach is used for the modeling of a sawmill workshop and the results are compared with those obtained previously by using only CART model or neural network model.
\end{abstract}

Keywords-Decision tree; CART; reduced model; neural network; simulation; supply chain; learning

\section{INTRODUCTION}

The decision making process in internal/external supply chains (SC) domain needs to evaluate planning or scheduling scenario. To do that, a simulation flow model is useful to highlight evolution of resources states, work in progress, and queues allowing to build a "predictive scheduling" [9].

This "predictive scheduling" may be called into question when significant events occur on the shop floor. This fact implies to perform a "reactive scheduling" by using information on these events collected by the real time systems [6]. The main difficulty is to exploit this information quickly in order to take decision $[12,13]$. The goal is to manage critical resource capacity which are mainly bottlenecks [20]. The theory of Constraints (ToC) consists in managing all the SC by bottlenecks control [4]. In this case, the evaluation of material flow by discrete events simulation model is useful [14]. However, the building of such models is a very complex task and lead to problem of scale [11] that is why different approaches of reduced/aggregated simulation modelling has been proposed [2, 3, 19]. Among these different approaches, the using of continuous flow models to approximate discrete manufacturing environments is one of the more investigated [19]. The authors have proposed and compared different types of continuous models including neural networks (NN) [19] or classification and regression trees (CART) [17]. These two tools are able to extract models directly from dataset. The main advantage of CART is that the resulting model is a sequential model which logically combines a sequence of simple tests. This fact allows to extract knowledge from the model. However, this type of model presents two main drawbacks: the adaptation of the model to system changes, and the weak stability of the model structure when different datasets are considered (two different datasets collected on the same system may lead to two very different models).

This paper investigates the association of NN and CART models in order to improve the continuous approximation of the reduced part of the simulation model. The main idea is to compare the structures of different CART models of the same system constructed with different datasets. The common part of these models is preserved when the divergent parts are discarded and replaced by NN models. This approach is tested and compared with the results obtained by using only $\mathrm{NN}$ or CART models on sawmill internal SC case.

The next section presents the reduced model building strategy. Part 3 is devoted to the considered industrial application case, when part 4 presents the results before to conclude.

\section{THE REDUCED MODEL}

\section{A. The algorithm}

The main idea of the approach is to determine which part (work centers) of the system is essential to model and which one is less important. This is performed according to the ToC concept [4] with the goal to reduce the number of elements, connections and model calculation in order to reduce complexity [22]. A literature review of reduction model approach can be found in [19]. The reduction model approach proposed here is based on the one proposed by Thomas and Thomas [18] where discrete event models and continuous models are associated to design the simulation model. The essential works centers of the model are described by using discrete event model when continuous model is used to determine how the essential work center are fed. The main 
steps of the algorithm are presented figure 1 where the first ones consist to determine which work centers (WC) need to be modelled with a discrete event model, considering the manufacturing order (MO) and which ones may be modelled with a continuous approximation. The last step (in gray) consists to design this continuous model and is the core of this paper.

For this step, the main idea is to exploit the normal production data to extract directly and automatically this model from the dataset. Different continuous models have been tested in the past including regression tree [17] which allows to extract knowledge form data and neural networks [18, 19] which are more adaptable to change of the system. This paper proposes to associate these two approaches. The main goals of this approach are to reduce the modelisation time by extracting automatically the continuous part of the model directly from the collected production dataset, and to reduce the computing time.

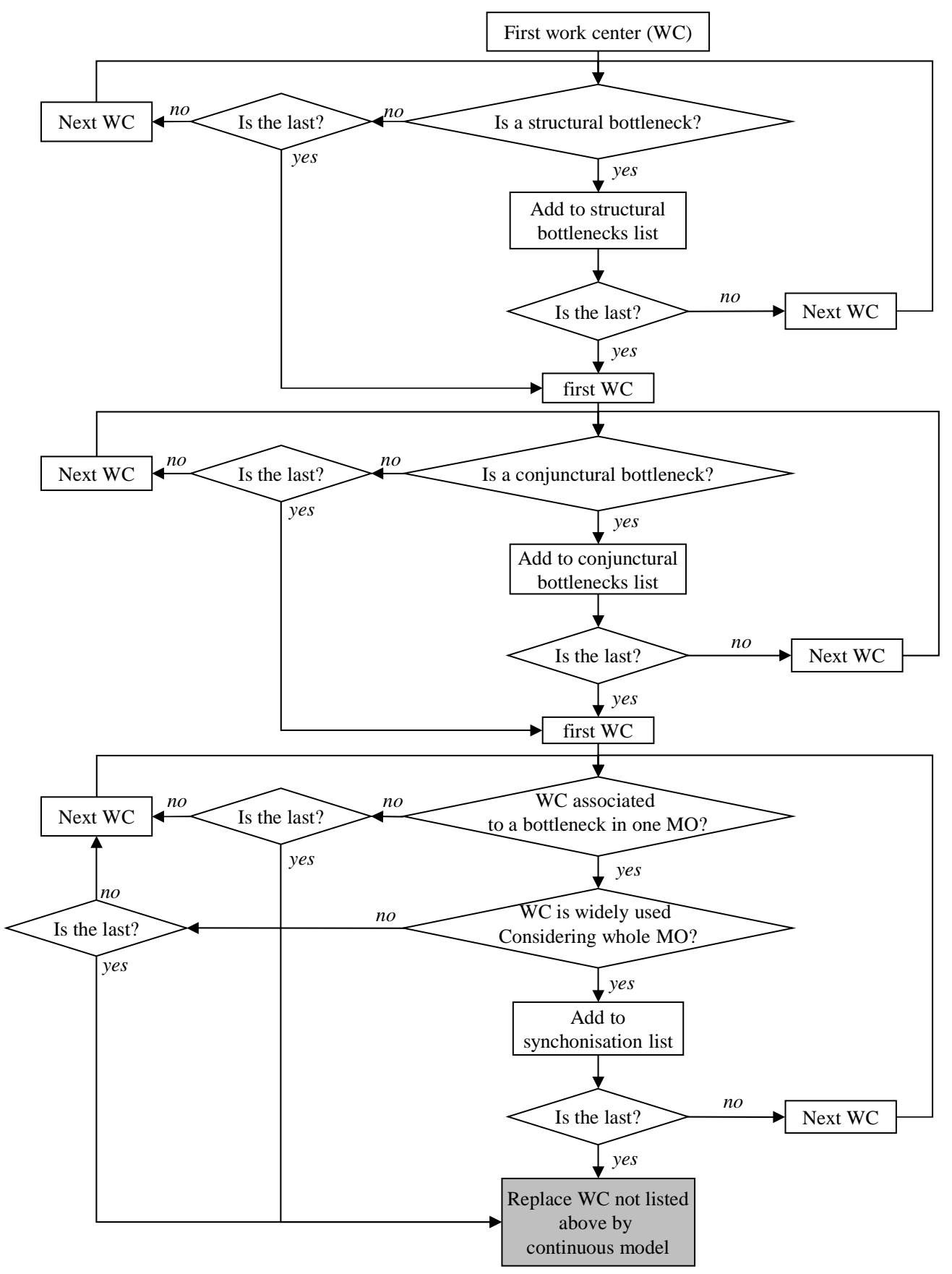

Figure 1. Reduction model algorithm 


\section{B. Regression tree}

Classification and regression trees are constituted by a sequence of simple logical tests. If in classification trees, the output corresponding to a pattern is the predicted class of the pattern, in regression tree, the output is the real value which is the more likelihood for the pattern and who will be the predicted value of the output [8]. The principle of regression tree is to split the dataset in two purer datasets at each node beginning from the root node until leafs. The goal is to find for each node the input to test and the value of the corresponding threshold in order to minimize the node impurities of its two children and for each leaf, the real value the more representative of the considering sub dataset.

Different algorithms have been proposed to design regression trees. The considered one, here, is the CART (Classification and Regression tree) which is based on the generalization of the binomial variance called Gini index [1].

The design of regression trees is a recursive procedure beginning from the root node which is stopped when only one pattern is associated to each leaf, or if the splitting of the subdataset becomes impossible due to the fact that each child node have identical distribution of predictor variable, or if the maximal number of levels in the tree fixed by designer is reached, or if an impurity improvement level threshold fixed by designer is reached.

Notwithstanding these stop criterions, overfitting problem occurs with resulting tree and a pruning step must be performed. This pruning step uses a penalty term representing the number of leafs associated to the same impurity term used during the design step (Gini). The final tree selection is performed by using a cross-validation procedure [1].

A regression tree allows to extract knowledge from dataset by giving a hierarchy into the influence of input variable considering the input variable associated to each node (the input variable associated to the test of the root node is probably more influent than the input variable associated to its child nodes).

However, the structure of the resulting model depends on the dataset and small variations in the dataset may lead to structure very different.

\section{Neural network}

Different artificial NNs have been proposed to solve many different problems: dynamic system identification, pattern classification, adaptive control... Among them, Multilayer perceptron (MLP) is the most popular [5]. Different activation functions (hyperbolic tangent, sigmoid, linear...) may be used for the neurons. In the considered structure, hidden neurons use hyperbolic tangent when output neuron exploits a linear one. The design of NN model is performed in three steps:

- Initialization consists to determine the initial set of weights and biases. The Nguyen Widrow algorithm is used [10],

- Training fits the network output to the data. The using of a robust Levenberg-Marquardt algorithm [15] allows to limit the impact of outliers and has a regularization effect.

- Pruning determines the optimal structure of the network and allows to avoid overfitting problem. The pruning procedure used is the one proposed by Thomas and Suhner [16].

\section{Proposed continuous model}

As explain previously, the structure of CART models depends on the dataset used. The main idea is to exploit the instability of the CART structure due to the difference between datasets in order to construct a shorter stable model.

The work is performed in three steps:

1. Constructing of diverse datasets by using bagging strategy.

2. Learning of diverse CART models on these diverse datasets.

3. Comparing the diverse CART models. For each node of the CARTs, a similarity measure is performed and compared to a threshold. If the similarity measure is higher to the threshold, the node is preserved, otherwise the node is discarded. When this procedure is completed for all nodes, a resulting CART model is obtain which includes only nodes which are mainly present in all CART models.

4. Replacing leafs of this resulting model by NN models. The learning of these different NN models is performed on the part of the dataset corresponding to the results of the tests performed by the ancestors of the considered leaf.

The value of the threshold used in step 3 is fixed here to $90 \%$ which signifies that only nodes belonging to more than $90 \%$ of the CART models are preserved.

\section{OVERVIEW OF THE SAWMILL}

To validate the proposed approach, a reduced simulation model of a sawmill workshop is built in order to help managers in their weekly decision-making Master Production schedule (MPS) process. The goal is to evaluate the effectiveness of the MPS, to maximize the global productivity of the bottleneck and so of the workshop, and to find the reason of some congestions of the trimmer WC.

A first study has been performed based on a complete model [14]. This study has allowed to find the bottleneck which is the last sawmill work center. Moreover, the reasons of unexplained congestion phenomena of the trimmer are identified, these congestion phenomena are related to a bottleneck load rate too high (higher than 60\%). Also these conditions degrade the bottleneck productivity and so must be avoided by taking the good decisions during the MPS process. However, all influent factors on bottleneck productivity depend on the first WC and so, a simulation flow model is essential for this decision making process. The complete simulation model built is not able to respond to this needing due to its prohibitive 
modification and computational time, so a reduced simulation model must be built.

In this section, the sawmill is described from a process point of view. By focusing on the material flows, two linear parallel flows may be identified for main and secondary products. These flows depend also of the variation of $\log$ dimensions. These facts implies that this process is clearly nonlinear.

This sawmill can be divided into three work centers. To describe the material flows, the trip of a log from its entrance to its exit (sawed in planks) is described. The Canter line (figure 2 ) is the first work center. The log is taken over by the input conveyors RQM1 to RQM3. A scanner MS determine its dimensions and in function of these dimensions, the $\log$ is driven to conveyors RQM4 or RQM5. These conveyors are used as input inventory for the Canter line. Two serial saws, the Canter machine and the CSMK saw, transform logs into square-shaped parallelepipeds. To shape the four sides of the parallelepiped, a production loops and a rotation of $90^{\circ}$ are needed by using RQM6, BT4, and BT5 conveyors and the log is stored in RQM7 which constitutes a work in process inventory. The MPS must determine which inventory (RQM4, RQM5, or RQM7) must fed the canter line. After the second passage of the log on the Canter machine, the squared is completed and four secondary products (two during the first passage and two during the second one) are taken out the Canter line by using BT4 or BT5 conveyors toward the second work center, the Kockum line. The parallelepiped continues on its road until the MKV saw where it is sawed into three planks (main products) which are conveyed to the third work center, the trimmer line.

The second work center (figure 3 ) is the Kockum line where the main machine is the Kockum saw. BT4 and BT5 are the two input conveyors for this line. Only secondary products are sawed by this work center. The product are sawed by QM11 and driven to the Kockum saw which optimizes the planks in accordance with the demand. These planks are finally driven to the third work center the trimmer line.
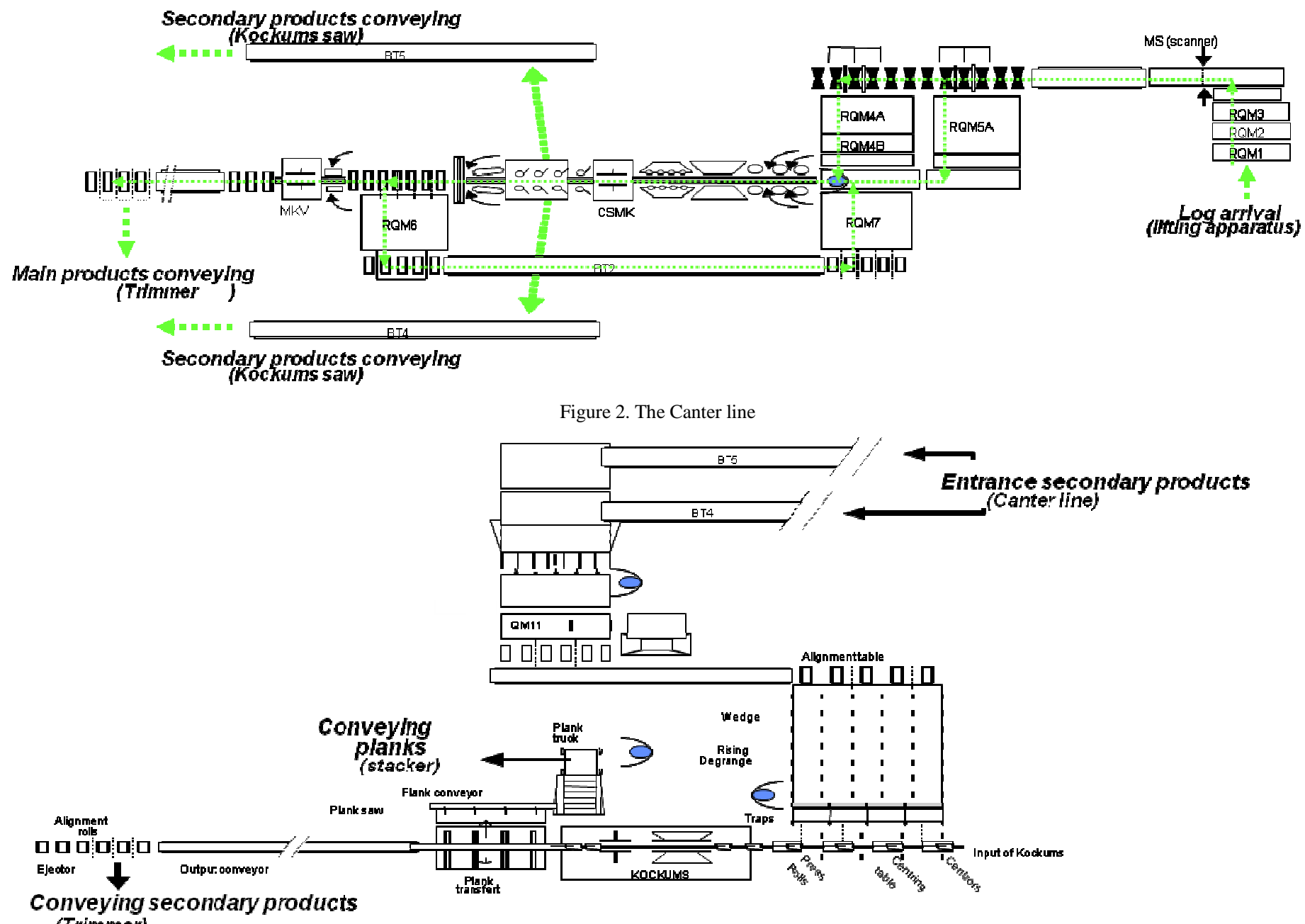

(Trimmer)

Figure 3. The Kockums line 

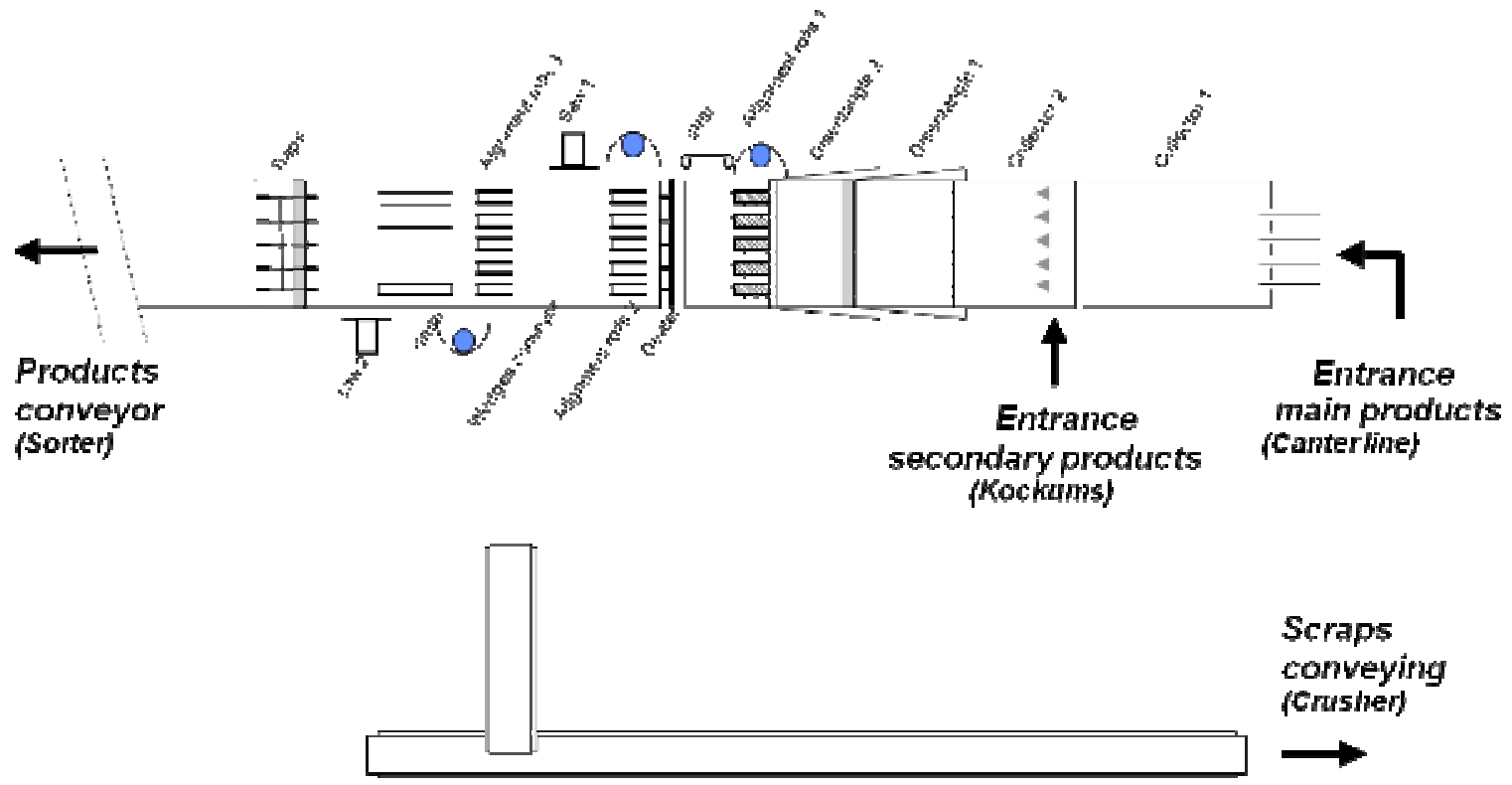

Figure 4. The trimmer line

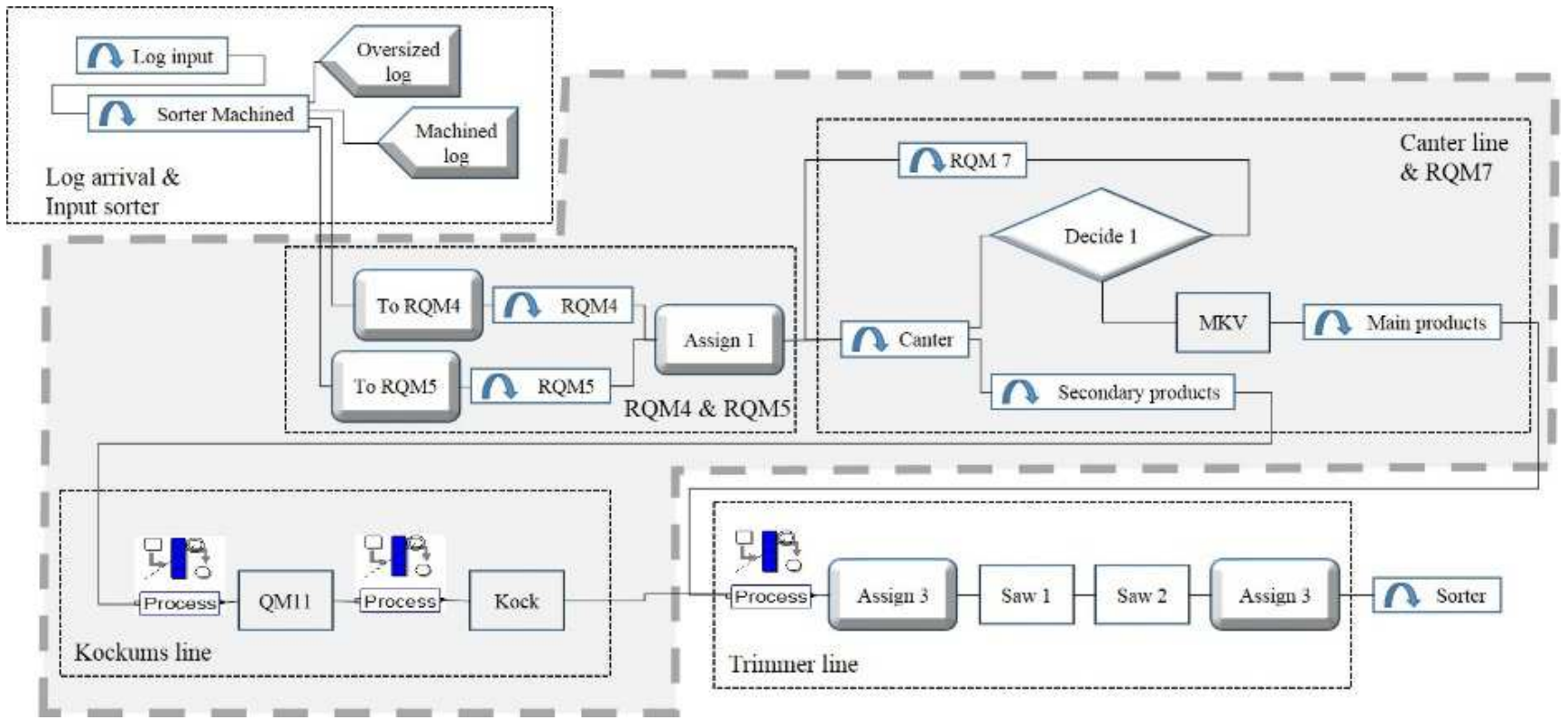

Figure 5. Complete model

The trimmer line (figure 4) is the third work center. First work [14] have shown that this is this work center is the bottleneck of the workshop. At this step, the flows of secondary products from the Kockum line (collector 2) and main products from Canter line (collector 1) come together. The trimmer line saw the products to length (Saw 2) and perform default bleeding (saw 1). The productivity of this work center depends to the decisions taken on the Canter line. This fact implies that the impact of bad decisions is see too late to be corrected.

\section{THE SIMULATION MODEL}

The bottleneck of the workshop is the last line (trimmer line). So, to reduce the model, a focus on this line may be performed and the precise modelling of the inventories (RQM4, RQM5, and RQM7) and of the Canter and Kockums work center with a discrete event model is unnecessary. Only a model able to determine how the bottleneck is fed is needed. So, in the complete model [14] presented figure 5, the model part surrounded in gray dashed line may be replaced by a continuous model which may be extracted automatically from the dataset. 
The first task to design this continuous model is to collect dataset and to identify the input variables [19]. These variables can be classified into three categories, variables related to products $(\log )$, process variables, and bill of material or routing variables (cutting plan).

The products variables are dimensional ones: length $(\lg )$, smallest (diaPB), greatest (diaGB) and mean (diaMOY) log diameters.

The process variables are collected at the arrival time of the $\log$. The input stock and the utilization rate of the bottleneck, (Q_trim, and U_trim), the number of works in progress present in the Canter work center (Q_RQM) and in the inventories (Q_RQM4, Q_RQM5 and Q_RQM7) are collected.

The routing variables correspond to the cutting plan variables which is here the type of products (T_piece).

In this paper, the complete model is used as benchmark, and the production of 12775 parts is simulated to construct the dataset.

The learning process of the tree or NN models is a supervised one. So the output variable must be identified and the corresponding data collected. The goal is to determine how the bottleneck is fed. So, we need to determine the throughput time $(\Delta \mathrm{T})$ of the products between their entry $(\log )$ in the Canter work center and their arrival in the input inventory of the bottleneck. These data are collected for the 12775 products simulated.

This dataset is used to fit the behavior of the reduced model to the complete one which serve as reference model.

To evaluate the performance of the learned continuous model, the dataset is randomly split into two datasets, one for the learning and one for the validation. To evaluate the distance between the model and the system, the Root Mean Square Error (RMSE) is used:

$$
R M S E=\sqrt{\frac{1}{N} \sum_{n=1}^{N}\left(y_{n}-\hat{y}_{n}\right)^{2}}
$$

where $N$ is the number of data, $y_{n}$ is the $n^{\text {th }}$ actual data point, and $\hat{y}_{n}$ is its predicted value.

A bagging strategy is used in order to construct 100 different datasets. These datasets are used to build 100 CART models. On these 100 CART models, only the common part (corresponding to the same tests performed by $90 \%$ of the CART models) is preserved to build a stable reduced CART model presented figure 6 .

In a second step, $9 \mathrm{NN}$ models are learned to replace the 9 leafs of the CART model. These $9 \mathrm{NN}$ models are learned on different datasets (subparts of the complete learning dataset with respect to the tests associated to the ancestors nodes of the considering leaf). These learning datasets are of length 759, $1893,518,656,730,974,580,680,107$ respectively when the validation is performed on datasets of length 732, 1782, 469, $660,731,974,620,712,101$ respectively. For each model, the learning is performed with less data than the complete dataset. This fact allows to reduce the computational time.

The results obtained with this model must be compared with those obtained with models build in preceding works, CART model [17] and NN model [19]. These results are presented in table 1 .

To evaluate the performance of the continuous model, the residual must be studied. First the residual must be a white noise and so its mean must be null. To do that, a two-tailed statistical hypothesis test must be used for the different algorithms.

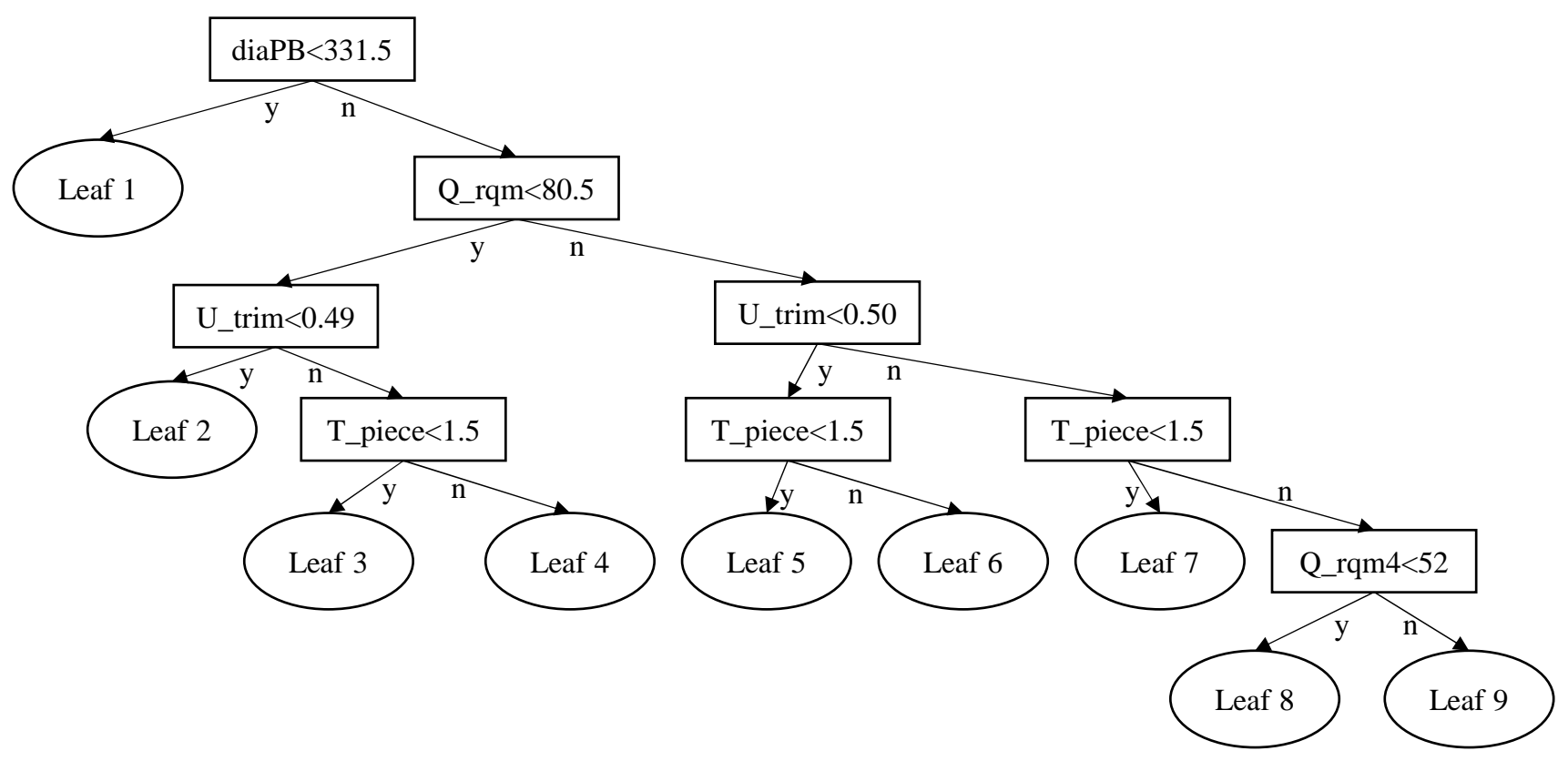

Figure 6. Reduced stable CART model 
The null hypothesis $\mathscr{H}_{0}$ (that the mean of the residuals is null) and its alternative $\mathscr{H}_{1}$ are:

$$
\begin{cases}\mathscr{H}_{0}: & \mu=0 \\ \mathscr{H}_{1}: & \mu \neq 0\end{cases}
$$

where $\mu$ is the mean of the residuals population. $\mathscr{H}_{0}$ is rejected with a risk level of $5 \%$ if:

$$
\left\{\begin{array}{l}
U=\frac{\bar{\varepsilon}}{s / \sqrt{N_{V}}}>1.96 \\
U=\frac{\bar{\varepsilon}}{s / \sqrt{N_{V}}}<-1.96
\end{array}\right.
$$

where $N_{V}$ is the size of the validation dataset, and $\bar{\varepsilon}, s^{2}$ are the estimated mean and variance of the residuals. The results of this test for the three compared models are presented table 1.

Moreover, the standard deviation of the residuals obtained with these models must compared in order to determine if the three models are significantly different.

For this case, the true standard deviation of the noise $\sigma_{0}$ is unknown. So, the goal is to determine if the results obtained with the two weakest models are significantly different to those obtained with the best one. So, in the statistical test, the true standard deviation of the noise $\sigma_{0}$ is supposed to be equal to the standard deviation of the residuals obtained with the best model.

The two-tailed statistical hypothesis test is used to determine whether the variance of the population $\sigma^{2}$ obtained with the different algorithms was statistically different to $\sigma_{0}^{2}$. The null hypothesis $\mathscr{H}_{0}$ and its alternative $\mathscr{H}_{1}$ were therefore:

$$
\begin{cases}\mathscr{H}_{0}: & \sigma^{2}=\sigma_{0}^{2} \\ \mathscr{H}_{1}: & \sigma^{2}>\sigma_{0}^{2} \\ & \sigma^{2}<\sigma_{0}^{2}\end{cases}
$$

and $\mathscr{H}_{0}$ can be rejected with a risk level of $5 \%$ if:

$$
\left\{\begin{array}{l}
\Gamma=\frac{\left(N_{V}-1\right) \cdot s^{2}}{\sigma_{0}^{2}}<\Gamma_{c 1}=\chi^{2}\left(v, \frac{\alpha}{2}\right) \\
\Gamma=\frac{\left(N_{V}-1\right) \cdot s^{2}}{\sigma_{0}^{2}}>\Gamma_{c 2}=\chi^{2}\left(v, 1-\frac{\alpha}{2}\right)
\end{array}\right.
$$

where $v$ is the number of degrees of freedom, and $\alpha$ is the confidence interval. For this case, the two bounds $\Gamma_{c 1}$ and $\Gamma_{c 2}$ were 7011 and 6555 . The results of this statistical hypothesis test on the validation datasets are presented in Table 1.

These results show that the three models give mean of residual statistically null $(-1.96<\mathrm{U}<1.96)$. However, the tree model outperforms the NN model on the identification dataset. On this dataset, there is no statistically significant difference between results obtained with tree model and proposed model.

On the validation dataset, the proposed model outperforms the two other with a statistical significance $(\Gamma$ values are outside of the bounds $\Gamma_{c 1}$ and $\Gamma_{c 2}$ ). So the proposed approach improves the results.

Considering the computational time, the proposed approach is clearly more time consuming than the two other and particularly to the tree model (more than 3 minutes comparing to less than 2 seconds for the tree model). This fact is mainly due to the pruning step performed on each of the $9 \mathrm{NN}$ sub models. However in case of adaptation of the model in case of change of the considered systems, this pruning step may be omitted because the optimal structure of the networks are already determined. Moreover, in this case, only the relearning of the NN models which present a drift with the considered system must be relearned and so, it is not necessary to perform this relearning work on the complete model on the contrary to the two other approaches.

TABLE I. RESULTS OBTAINED WITH THE THREE MODELS

\begin{tabular}{|c|c|c|c|c|c|}
\cline { 2 - 6 } & \multicolumn{2}{|c|}{ RMSE } & \multicolumn{2}{c|}{ Statistical tests } & \\
\cline { 2 - 6 } & Identification & Validation & $\mathrm{U}$ & $\Gamma$ & Computing time (s) \\
\hline NN model & 408,45 & 413,93 & $7.28^{*} 10^{-4}$ & 12272 & 107.277 \\
\hline Tree model & 263,47 & 324,10 & $1.21^{*} 10^{-4}$ & 7533 & 1.621 \\
\hline Tree-NN model & 268.7189 & 308.4844 & $8.96^{*} 10^{-4}$ & - & 184.74 \\
\hline
\end{tabular}




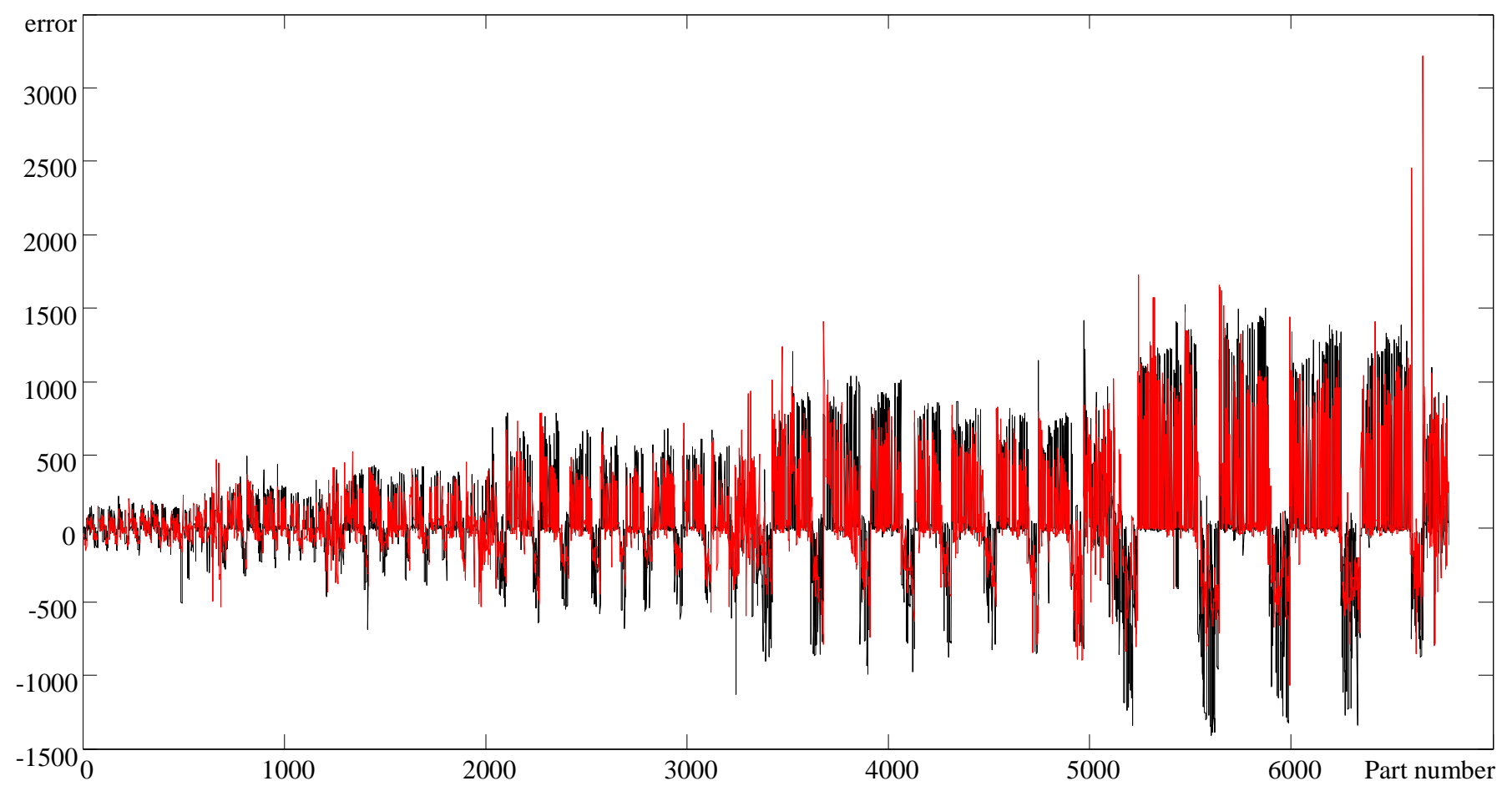

Figure 7. Error performed by the tree model (black) and the proposed one (red)

The figure 7 presents the error obtained with the tree model (in black) and the proposed model (in red). This figure shows that the proposed models allows to improve the prediction and limits the size of the great errors.

Moreover, the proposed model may easily translated into a discrete event model using a tool like Arena@. This translation is presented figure 8 . The nodes of the tree part of the model are represented by "decide blocs" when leafs which correspond to the neural models may be described by using "VBA blocs".

\section{CONCLUSION}

The association of tree and neural networks models to build reduced simulation model is investigated here. In a first step tree models are built on different datasets. These models are compared and the different nodes are discarded. The resulting leafs are replaced by using neural networks.

This resulting model is used to model the functioning of a part of the process that is not a bottleneck in order to determine how the bottleneck is fed. This approach is applied to the modelling of a sawmill workshop.

The results show that reduced model is efficient to represent the material flows. The comparison of the results obtained with the proposed approach with those obtained with only tree model or neural network model shows that the proposed approach outperforms the two others. Moreover, the modularity of the resulting model allows to simplify its adaptation in case of change on the real system.

At last, the design of the continuous model is performed by using knowledge discovery in data process with may be partially automated. The main task of the modeler is to determine which variables must be collected and to prepare the dataset. So, the design of a reduced model is a faster and easier task because modeler can focus on the bottleneck model. 


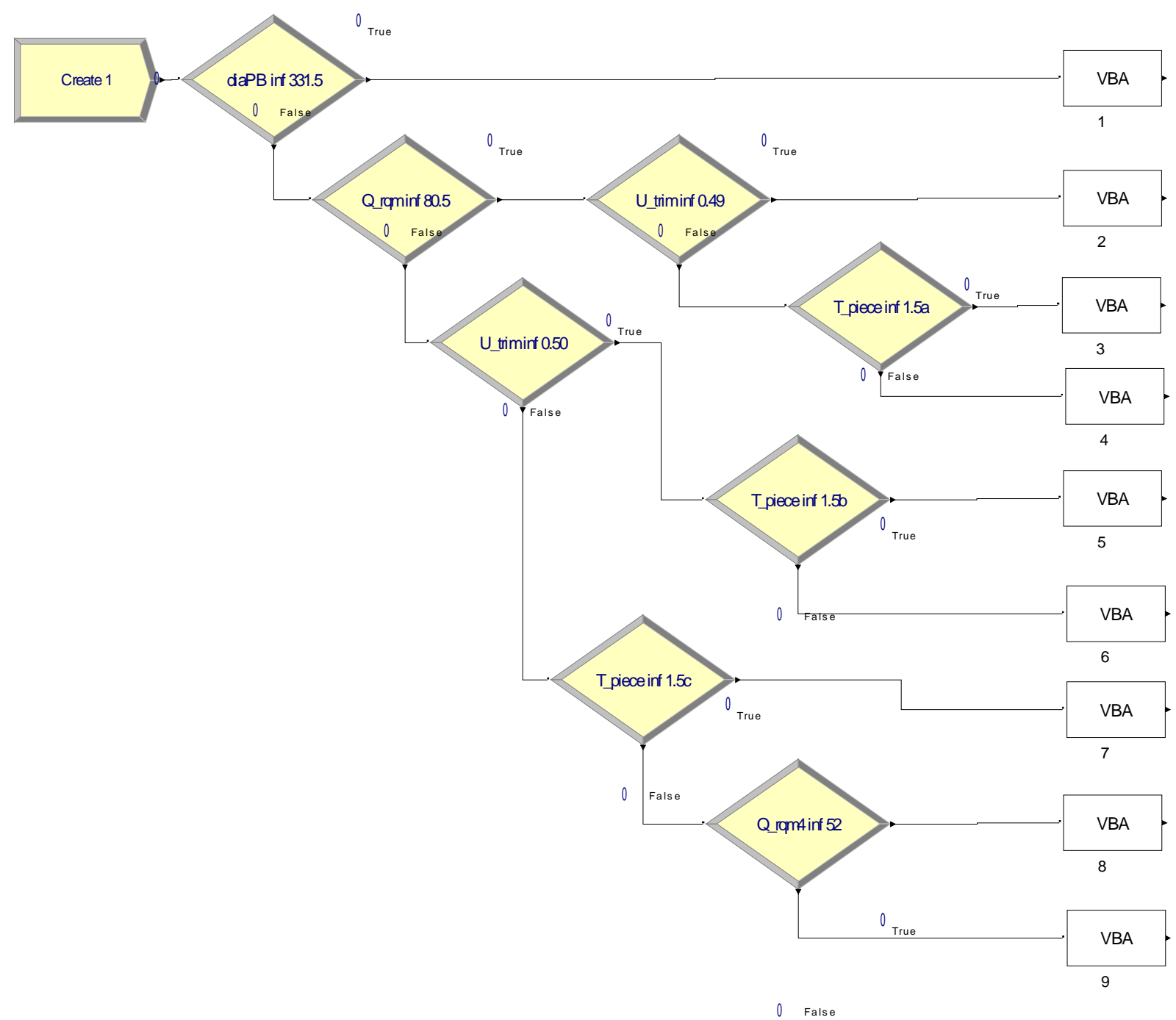

Figure 8. Translation of model in ARENA@

\section{REFERENCES}

[1] Breiman L., Friedman J.H., Olshen R.A., Stone C.J., 1984. Classification and regression trees, Chapmann \& Hall, Boca Raton, USA.

[2] Brooks, R.J., Tobias, A.M., 2000. "Simplification in the simulation of manufacturing systems". International Journal of Production Research, 38(5): 1009-1027.

[3] Chwif, L., Paul, R.J., Pereira Barretto, M.R., "2006. Discret event simulation model reduction: A causal approach". Simulation Modelling Practice and Theory, 14: 930-944.

[4] Goldratt., E., Cox, J., 1992. The Goal: A process of ongoing improvement, North River Press; 2nd Revised edition, Great Barrington, USA.

[5] Han, H.G., Qiao, J.F., 2013. "A structure optimisation algorithm for feedforward neural network construction". Neurocomputing 99: 347357.

[6] Khouja, M., 1998. "An aggregate production planning framework for the evaluation of volume flexibility". Production Planning and Control, 9(2), 127-137.
[7] Lewis R.J., 2000. "An introduction to classification and regression tree (CART) analysis". Annual Meeting of the Society for Academic Emergency Medicine, San Francisco, California, May 22-25

[8] Loh, W.Y., 2011. "Classification and regression trees", Wiley Interdisciplinary Reviews: Data Mining and Knowledge Discovery, 1, 14-23.

[9] Lopez, P., and Roubellat, F. 2001. Ordonnancement de la production, Hermès, Paris.

[10] Nguyen, D., Widrow, B., 1990. «Improving the learning speed of 2layer neural networks by choosing initial values of the adaptive weights ». Proc. of the Int. J. Conf. on Neural Networks IJCNN'90 3: 2126.

[11] Page, E.H., Nicol, D.M., Balci, O., Fujimoto, R.M., Fishwick, P.A., L'Ecuyer, P., and Smith, R., 1999. "An aggregate production planning framework for the evaluation of volume flexibility". Winter Simulation Conference, 1509-1520.

[12] Pritsker, A., and Snyder, K., 1994. "Simulation for planning and scheduling", APICS, August.

[13] Roder, P., 1994. "Visibility is the key to scheduling success", APICS Planning and Scheduling, August 
[14] Thomas, A., Charpentier, P., 2005. "Reducing simulation models for scheduling manufacturing facilities". European Journal of Operational Research, 161(1): 111-125.

[15] Thomas, P., Bloch, G., Sirou, F., Eustache, V. 1999. "Neural modeling of an induction furnace using robust learning criteria". Journal of Integrated Computer Aided Engineering 6: 15-25.

[16] Thomas, P., Suhner, M.C., 2014. "A new multilayer perceptron pruning algorithm for classification and regression application". Neural Processing Letters, DOI 10,1007/s11063-014-9366-5.

[17] Thomas, P., Suhner, M.C., Thomas, A. 2014. "CART for Supply Chain Simulation Models Reduction: Application to a Sawmill internal Supply Chain". International Conference Advances in Production Management Systems APMS'14, Ajaccio, France, September 2024.
[18] Thomas, P., Thomas, A., 2011. "Multilayer Perceptron for Simulation Models Reduction: Application to a Sawmill Workshop". Engineering Applications of Artificial Intelligence, 24, 646-657.

[19] Thomas, P., Thomas, A., Suhner, M.C., 2011. "A neural network for the reduction of a Product Driven System emulation model". Production Planning and Control, 22, 767-781.

[20] Vollmann, T.E., Berry, W.L., Whybark D.C., 1992. Manufacturing, Planning and Systems Control. The Business One Irwin.

[21] Ward, S.C., 1989. "Argument for constructively simple models". Journal of the Operational Research Society, 40(2): 141-153.

[22] Zeigler, B.P., 1976. Theory of modelling and simulation. Wiley, New York. 